\title{
Effects of 2.0MPa Heliox Exposure on Working Memory of Divers
}

\author{
Jiali Wang* and Lvqing Miao \\ Institute of Nautical Medicin, Nantong University, Nantong, Jiangsu, China \\ *Corresponding author
}

\begin{abstract}
Objective: To study the effects of 2.0Mpa heliox exposure on Working Memory of professional divers. Methods: Four divers participated in this study. Performances on kinesthetic memory, Visual Spatial Working Memory and Object Working Memory were examined before heliox saturation, 2.0MPa heliox saturation and decompressed. Results: Repeated measures ANOVA showed that divers' performances on kinesthetic memory were not statistically significant, but the direction of errors was opposite. The RT of visuo-spatial working memory and the ACC and RT of object Working Memory were statistically significant $(F=5.756, \quad P<0.05$. Conclusion: $2.0 \mathrm{Mpa}$ heliox exposure has an influence on working memory.
\end{abstract}

Keywords-2.0Mpa heliox exposure; kinesthetic memoryj; visual spatial working memory; object working memory

\section{INTRODUCTION}

In saturation diving, divers spend a long time staying under pressure after most of their tissues are saturated with breathing gas, like heliox. Exposure to a hyperbaric environment may leads to a series change in inflammatory cytokines (Tian et al.,2014; Matsuo et al., 2000; Krog et al., 2010), neuroendocrine (McLellan et al., 2010; Hirayanagi et al., 2003), extreme tiring experience of body, sleep disruption (Nagashima et al., 2002), and the psychological stress in mind (Curley et al., 1979; Biersner et al., 1984), resulting in alterations of divers' mood, cognition and performance efficacy, which are critical for diving work.

Some researchers studied the effect of deep water diving on cognitive ability and performance efficacy. The results are not consistent. Hamilton found that $360 \mathrm{~m}$ helium-oxygen saturation diving did not affect the finger flexibility of divers (Hamilton,1976); while another study reported increased finger tremors at 485m diving (especially after304m) in six divers (Berghageetal.,1975). Most of studies focused on the associative memory, short-term memory, arithmetic ability, perception, mental rotation, hand-eye coordination and grip strength, Few, if any, studies have attempted to illustrate the effect on working memory of saturation diving, Working memory(WM) generally be defined as the cognitive system responsible for maintaining information or task goals in an active state over brief periods of time.WM has become central to understanding complex cognition and have been linked to various higher level cognition, including reading comprehension, executive function, reasoning abilities, learning and general fluid intelligence(McCabe,2010;Zhao et al.,2010; Eriksson et al.,2015).
In this study, we investigated the effect of 2.0MPa heliox exposure on working memory, and to give a view on how high pressure influences the cognitive abilities.

\section{METHODS}

\section{A. Subjects}

Four male professional divers aged from 24-35 years old recruited from Shanghai Salvage Department of Ministry of Transport of the People's Republic of China. Physical and mental health examination showed they are all healthy 。

The study is approved by ethic committee of human study in Nantong University and all subjects provided written consent for experimental procedures. The procedures followed the guidelines of human research from ethic committee in Nantong University.

\section{B. Materials and Experiments}

Experiment1: kinesthetic memory

Kinesthetic orientation tester was used to test the divers ability of kinesthetic memory. First, the subjects were kept blindfolded to experience with a certain angular degree $(30,70,90)$ using their arm, then they were asked to find the angular degree blindfold. Each of three angular degree was repeated three times and the average was taken.

\section{Experiment2: Visual Spatial Working Memory}

In this part, 2-back task was used. Subjects were asked to judge whether the location of stimuli displayed in current screen is the same to one interval former one. Experimental procedure was designed by E-prime2.1, and presented by Lenovo-G50

\section{Experiment3}

This part was similar to experiment2, only object stimuli were constituted for spatial stimuli.

\section{Simulation of Saturation Diving}

The simulation was conducted at the diver living chamberof $200 \mathrm{~m}$ saturation diving system at Institute of Nautical medicine, Nantong University.The chamber has an internal diameter of $2.24 \mathrm{~m}$ and length $5.5 \mathrm{~m}$ and living volume15.2m3. The chamber is filled with helium-oxygen, temperature $29-31^{\circ} \mathrm{C}$, moisture $50-70 \%$, noise lower than $60 \mathrm{~dB}$ and light intensity 50-100lx. 
The study was divided into before heliox saturation, 2.0MPa heliox saturation and decompressed. For all tests presented above, the divers were trained repeatedly to reach a stable baseline, and the last score was taken as baseline.

\section{Statistics}

Repeated variance analysis and LSD post hoc test was conducted with SPSS17.0 software(Chicago,IL,USA) .

\section{RESULTS}

\section{A. Kinesthetic Memory}

Repeated variance analysis for error of Kinesthetic memory showed that there is no significant differences among three groups( $\mathrm{F}=0.834, \mathrm{P}>0.05$, Table1).

\section{B. Visual Spatial Working Memory}

- For the ACC of visual spatial working memory, Repeated variance analysis showed there is no significant difference among three groups. (Table2), but showed significant difference in RT ( $\mathrm{F}=5.756$, $\mathrm{P}<0.05$ ) , effect size 0.451 (Table3). LSD post hoc test revealed that before heliox saturation and $2.0 \mathrm{MPa}$ heliox saturation, 2.0MPa heliox saturation and decompressed group have difference significantly ( $\mathrm{P}=0.046,0.006$ respectively ), whereas before heliox saturation and decompressed groupshowed no significant difference $(\mathrm{P}>0.05)$.

\section{Object Working Memory}

Repeated variance analysis showed that the RT of object working memory among three groups was significant different ( $\mathrm{F}=19.091, \mathrm{P}=.022$, Table4). LSD post hoc test revealed that before heliox saturation and decompressed group, before heliox saturation and decompressed group have difference significantly ( $\mathrm{P}=0.042,0.022$ respectively ) , whereas 2.0MPa heliox saturation and decompressed group showed no significant difference $(\mathrm{P}>0.086)$.

For the ACC of object working memory, there is significant difference among three groups $(\mathrm{F}=189.00, \mathrm{P}=.000$, Table5). LSD post hoc test revealed that before heliox saturation and decompressed group, 2.0MPa heliox saturation and decompressed group, before heliox saturation and $2.0 \mathrm{MPa}$ heliox saturation all have difference significantly $(\mathrm{P}<0.01)$.

\section{DISCUSSION}

Extreme environment stress has been known to induce changes not only in the body but also the mind, resulting in various cognitive ability decrease(Yuan and Hou, 2015; Yuan et al., 2015, Hirayanagi et al., 2003; Brubakk et al.,).In present study with simulated helium-oxygen saturation diving experiment, we found that the error of Kinesthetic memory and ACC of visual spatial working memory were no differences among before heliox saturation , 2.0MPa heliox saturation and decompressed, the RT of visual spatial working memory, ACC and RT of object working memory were significantly different, suggesting that exposure to 2.0Mpa heliox saturation may impair the working memory of divers. Because working memory is regarded as the central and basic of higher cognitive function, we may speculate that working memory impairment is the essential reason for various decreases in cognition and performance efficacy, if so, we can train the working memory of divers to prevent that decrease.Although Post hoc test showed that ACC and RT of visual spatial working memory and RT of object working memory had no differences between 2.0MPa heliox saturation and decompressed group, the ACC of object working memory showed significant difference among three groups, suggesting that even decomposed to normal atmosphere pressure, the effect of heliox exposure cannot dismiss immediately.

In addition, we found that even the error of kinesthetic memory showed no difference among three groups but the direction of the error was opposite. The error of kinesthetic memory was negative before heliox saturation and decomposed, positive when at 2.0MPa heliox Saturation. Whether it is due to heliox saturation need further experiment to testify.

TABLE I. KINESTHETIC MEMORY ACROSS DIFFERENT PHASES OF THE EXPERIMENT.

\begin{tabular}{cccccc}
\hline & Mean & SD & F & P & $\begin{array}{c}\text { Partial Eta } \\
\text { Squared }\end{array}$ \\
\hline before & -5.639 & 1.030 & & & \\
2.0Mpa & 4.848 & 0.955 & 0.834 & 0.423 & .071 \\
$\begin{array}{c}\text { decompress } \\
\text { ed }\end{array}$ & -4.314 & 0.639 & & & \\
\hline
\end{tabular}

TABLE II. ACC OF VISUAL SPATIAL WORKING MEMORY.

\begin{tabular}{cccccc}
\hline & Mean & SD & F & P & $\begin{array}{c}\text { Partial Eta } \\
\text { Squared }\end{array}$ \\
\hline before & .850 & .043 & & & \\
2.0 Mpa & .808 & .023 & 1.339 & .291 & .161 \\
decompressed & .874 & .019 & & & \\
\hline
\end{tabular}

TABLE III. RT OF VISUAL SPATIAL WORKING MEMORY.

\begin{tabular}{cccccc}
\hline & Mean & SD & F & P & $\begin{array}{c}\text { Partial Eta } \\
\text { Squared }\end{array}$ \\
\hline before & 505.082 & 61.549 & & & \\
$2.0 M p a$ & 575.854 & 80.198 & 5.756 & .022 & .451 \\
$\begin{array}{c}\text { decompresse } \\
\text { d }\end{array}$ & 550.810 & 64.083 & & & \\
\hline
\end{tabular}


TABLE IV. RT OF OBJECT WORKING MEMORY.

\begin{tabular}{cccccc}
\hline & Mean & SD & F & P & $\begin{array}{c}\text { Partial Eta } \\
\text { Squared }\end{array}$ \\
\hline before & 495.460 & 5.822 & & & \\
2.0 Mpa & 527.600 & 11.054 & 19.091 & .022 & .864 \\
$\begin{array}{c}\text { decompresse } \\
\text { d }\end{array}$ & 508.345 & 4.267 & & & \\
\hline
\end{tabular}

TABLE V. ACC OF OBJect WORKING MEMOR.

\begin{tabular}{cccccc}
\hline & Mean & SD & F & P & $\begin{array}{c}\text { Partial Eta } \\
\text { Squared }\end{array}$ \\
\hline before & 95.0000 & 3.55903 & & & \\
& & & & & \\
2.0 Mpa & 89.0000 & 3.36650 & 189.00 & .000 & .984 \\
$\begin{array}{c}\text { decompresse } \\
\text { d }\end{array}$ & 91.2500 & 3.59398 & & & \\
\hline
\end{tabular}

\section{ACKNOWLEDGMENTS}

This work was supported by Project of Mental Health Protection for Commercial Divers Granted by Ministry of Transport of the People's Republic of China. We would also like to appreciate the divers and all staff taking part in this study.

\section{REFERENCES}

[1] Brubakk,A.O.,Ross,J.A.,andThom,S.R. (2014).Saturation diving; physiology and pathophysiology. Compr.Physiol. 4, 1229-1272.

[2] Hirayanagi,K.,Nakabayashi,K.,Okonogi,K.,andOhiwa,H.(2003).Autono mic nervous activity and stres shormones induced by hyperbari csaturation diving. Undersea Hyperb.Med. 30, 47-55. J. Clerk Maxwell, A Treatise on Electricity and Magnetism, 3rd ed., vol. 2. Oxford: Clarendon, 1892, pp.68-73.

[3] Hou, G.,Zhao,Y.,Yang,X.,andYuan,T.F.(2015). Autophagy does not lead to the asymmetrical hippocampal injury in chronic stress. Physiol.Behav. 144, 1-6. K. Elissa, "Title of paper if known," unpublished.

[4] Lewisa, V. J., \& Baddeley, A. D. (1981). Cognitive performance, sleep quality and mood during deep oxyhelium diving. Ergonomics, 24(10), 773-793.

[5] Nagashima,H.,Matsumoto,K.,Seo,Y.,Mohri,M.,Naraki,N.,and Matsuoka,S. (2002).Sleep patterns during 30-m nitrox saturation dives and in a confined atmospheric environment. PsychiatryClin.Neurosci. 56, 267-268.

[6] Yuan,T.F.,and Hou,G.(2015).The effects of stress on glutamatergic transmission in the brain. Mol.Neurobiol. 51, 1139-1143.

[7] Yuan,T.F.,Hou,G.,andArias-Carrion,O.(2015).Chronic stress impacts on olfactory system. CNS Neurol.Disord.DrugTargets 14, 486-491.

[8] Vaernes R, Bennett P B, Hammerborg D, et al. Central nervous system reactions during heliox and trimix dives to $31 \mathrm{ATA}[\mathrm{J}]$. Undersea biomedical research, 1982, 9(1): 1-14. 